\title{
POSTNATAL GROWTH IN PRETERM INFANTS FED PROBIOTICS : PREMAPRO STUDY
}

S. Hays ${ }^{1}$, A. Jacquot ${ }^{2}$, H. Gauthier ${ }^{3}$, E. Jumas-Bilak ${ }^{4}$, A. Beissel ${ }^{3}$, O. Pidoux ${ }^{2}$, E. Lachambre ${ }^{5}$, F. Rochat ${ }^{6}$, G. Cambonie $^{2}$, O. Claris ${ }^{3}$, J.-C. Picaud ${ }^{1}$, PREMAPRO Study Group

${ }^{I}$ Neonatologie, Hopital de La Croix Rousse, Lyon, ${ }^{2}$ Neonatologie, Hopital Arnaud de Villeneuve, Montpellier, ${ }^{3}$ Neonatologie, Hopital Femme-Mere-Enfant, Bron, ${ }^{4}$ Bacteriologie EA 3755 UM1, Faculté de Pharmacie, Montpellier, ${ }^{5}$ Nestle France, Noisiel, France, ${ }^{6}$ Nestec, Lausanne, Switzerland

Background: Significant reduction of necrotizing enterocolitis (NEC) and mortality rate has been recently reported in preterm infants supplemented with oral probiotics.

Aim: To evaluate postnatal growth in preterm infants supplemented with different probiotic preparations and the occurence of late onset sepsis.

Population and methods: Prospective, randomized, double-blind, controlled study performed in 3 tertiary care neonatal units in France (Lyon, Montpellier). Preterm infants (26-31wks, 700-1600g) were randomly designed to receive daily supplementation with placebo $(\mathrm{C})$ or probiotics $(\mathrm{P})$ : bifidobacterium lactis $(\mathrm{P} 1)$, bifidobacterium longum (P2) or both (P3) during 4-6 weeks. The number of subjects needed to show a $200 \mathrm{~g}$ difference in body weight at the end of supplementation period, with a power of $90 \%$, was 46 per group. Aerobic and anaerobic blood cultures were performed when a late onset sepsis was suspected.

Results: 197 preterm infants (GA:29.1 \pm 14 wks, BW:1173 $\pm 210 \mathrm{~g}$ ) were included: Placebo ( $\mathrm{n}=52)$, Probiotics $(n=142)$ from the first week of life $(D O L=6.6 \pm 1.5 \mathrm{~d})$. At the end of the supplementation period, there was no difference in body weight (group $\mathrm{C}=1906 \pm 23 \mathrm{~g}$, group $\mathrm{P}=1875 \pm 14 \mathrm{~g}, \mathrm{p}=0.25$ ) length and head circumference. The incidence of NEC was very low and similar between the 2 groups (NS). The incidence of late onset sepsis was similar between the 2 groups. None of these sepsis was related to a bifidobacteria. At term corrected age, there was no difference in anthropometric measurements and body composition analysis.

Conclusion: Preterm infants supplemented with bifidobacteria did not exhibit a better postnatal growth. No adverse effect was related to probiotics. 